\title{
Pareto optimality solution of the Gauss-Helmert model
}

\author{
B.Paláncz \\ Department of Photogrammetry and Geoinformatics \\ Budapest University of Technology and Economy, H-1521, Hungary \\ e-mail: palancz@epito.bme.hu \\ J. L. Awange \\ Department of Spatial Sciences \\ Curtin University, Australia \\ e-mail: j.awange@curtin.edu.au \\ L. Völgyesi \\ Department of Geodesy and Surveying \\ Budapest University of Technology and Economy, H-1521, Hungary \\ e-mail: volgyesi@eik.bme.hu
}

\begin{abstract}
The Pareto optimality method is applied to the parameter estimation of the GaussHelmert weighted 2D similarity transformation assuming that there are measurement errors and/or modeling inconsistencies.

In some cases of parametric modeling, the residuals to be minimized can be expressed in different forms resulting in different parameter values for the estimated parameters. Sometimes these objectives may compete in the Pareto sense, namely a small change in the parameters can result in an increase in one of the objectives on the one hand, and a decrease of the other objective in the other hand. In this study, Pareto optimality approach was employed to find the optimal trade-off solution between the conflicting objectives and the results compared to those from ordinary least squares (OLS), total least squares (TLS) techniques and the least geometric mean deviation (LGMD) approach.

The results indicate that Pareto optimality can be considered as their generalization since the Pareto optimal solution produces a set of optimal parameters represented by the Pareto-set containing the solutions of these techniques (error models). From the Pareto-set, a single optimal solution can be selected on the basis of the decision maker's criteria. The application of Pareto optimality needs nonlinear multi-objective optimization, which can be easily achieved in parallel via hybrid genetic algorithms built-in engineering software systems such as Matlab. A real-word problem is investigated to illustrate the effectiveness of this approach.
\end{abstract}

\section{Keywords}

Pareto optimality, Gauss-Helmert transformation, parameter estimation, measurement and modeling errors, least squares approach, genetic algorithm.

\section{Introduction}


Parameter estimation of coordinate transformations is a central problem in geodesy, and requires the handling of overdetermined systems of nonlinear equations containing more equations $(n)$ than unknown parameters $(m)$

$$
y_{i}=g_{i}(x, p)+e_{y_{i}}, \quad i=1,2, \ldots, n,
$$

which includes a parameter vector $p$ of dimension $m$, as unknown, and observation vectors $x$ and $y$. In this model, the underlying assumption is that the observation vector $x$ is error-free, and only $y$ has measurement errors $e_{y}$. This traditional error model can be solved in the least squares sense by using the ordinary least squares method (OLS).

However, this model is often invalid as many physical systems encounter errors in both the observation vectors, arising from, e.g., imprecise measuring instruments, human errors, etc. In such cases, the deviation between the model and the measured variables can be expressed by the compensation in the input variables, too

$$
y_{i}=g_{i}\left(x-e_{x}, p\right)+e_{y_{i}}, \quad i=1,2, \ldots, n .
$$

This model representing errors in both input as well as in output variables is called errors-in-all-variables (EIV) model whose parameter estimation problem leads to a minimization

$$
\min _{p, e}\left(e^{T} \cdot e\right)
$$

under the constraint of Eq. (2), where $e=\left(\begin{array}{l}e_{y} \\ e_{x}\end{array}\right)$. This is a nonlinear problem even if the model $(g)$ is linear, since now the errors $(e)$ are also unknowns besides the parameters $(p)$.

One of the frequently employed techniques to solve this parameter estimation problem is the so called total least squares method (TLS), see e.g. Golub and Van Loan (1980). Since this technique is basically designed for linear cases, one needs to linearize the original problem. For the linear Gauss-Helmert $(\mathrm{GH})$ transformation, the EIV problem has been solved e.g., by Felus and Schaffrin (2005) employing the TLS technique. For weighted coordinates, namely

$$
\min _{p, e}\left(e_{y}, e_{x}\right)\left(\begin{array}{cc}
w_{y} & 0 \\
0 & w_{x}
\end{array}\right)\left(\begin{array}{l}
e_{y} \\
e_{x}
\end{array}\right),
$$

Neitzel (2010) discussed the solution suggesting an iteratively linearized TLS (IL TLS) method.

The EIV model assumes that there are no modeling inconsistencies, namely the $y=g(x, p)$ model would be adequate, error-free if there were no measurement errors. This assumption is represented by Eq. (2).

In order to avoid this supposition and taking into account possible modeling errors, Tofallis (2002) and (2003) introduced a different approach to solve the EIV problem. He considered a simple line fitting, assuming that $x, y \in R^{1}$ and $p \in R^{2}$. Then Eq (1) becomes

$$
y_{i}-e_{y_{i}}=x_{i} p_{1}+p_{2}, \quad i=1,2, \ldots, n .
$$


Now the deviation between the measurements and the model can be expressed by the compensation of the input too, namely

$$
y_{i}-e_{y_{i}}=\left(x_{i}-e_{x_{i}}\right) p_{1}+p_{2}, \quad i=1,2, \ldots, n .
$$

He then proposed to minimize the sum of the squares of the geometric means of the errors,

$$
\min _{p_{1}, p_{2}} \sum_{i=1}^{n}\left(\sqrt{\left|e_{x_{i}} e_{y_{i}}\right|}\right)^{2} .
$$

This technique is referred as least geometric mean deviation (LGMD) method.

To find another solution technique for the EIV problem of the GH model, let us express the squares of the errors considering Eqs (5) and (6) as,

$$
f_{1}(p)=e_{y}^{T} e_{y}
$$

and

$$
f_{2}(p)=e_{x}^{T} e_{x} .
$$

Realizing that these functions are competing such that one increases at the expense of the other, Pareto optimality can be employed as an alternative method.

In this study, an extension of this approach for the $\mathrm{GH}$ model with weighted coordinates will be illustrated and the results compared to those of the IL-TLS, as well as, to those of the LGMD method.

The study is organized as follows: Section 2 provides a brief outline of the Pareto optimality approach while Section 3 considers, the parameter estimation problem of the 2D similarity transformation $(\mathrm{GH})$ as a multiobjective minimization problem. In Section 4, a numerical example illustrates the suggested approach. Section 5 compares the different parameter estimation techniques and Section 6 concludes the features of the Pareto optimality solution by contributing to the solution of a real-world 2D datum transformation problem of Hungarian geodetic stations covering an area of $70 \times 70 \mathrm{~km}$.

\section{Pareto optimality}

In many real-life situations, there are multi-objective optimality problems, which means that there is more than one objective to be minimized or maximized. In cases where all of the objective functions increase or decrease, there exists no optimum. However, in regions where these objective functions are competing or conflicting with each other, meaning that a small change in the independent variables will result in an increase of one objective function, an optimum can exist. We call such regions feasible regions for optimal solutions.

A solution in such region is said to be a Pareto optimal solution if it is not dominated by any other solution in that region. Pareto optimality is defined as follows, see e.g. Marler and Arora (2004):

Definition: A point, $x^{*} \in X$, is Pareto optimal if there exists no other point, $x \in X$, such that $f(x) \leq f\left(x^{*}\right)$ and $f_{i}(x)<f_{i}\left(x^{*}\right)$ for at least one function. 
From the above definition, the Pareto optimal solution is therefore a set of solutions, rather than a single one. The independent variables representing these solutions in the variable space form a Pareto-set, and the corresponding values of the objective functions are labeled as the Pareto-front. In our case the objective functions are convex therefore the Pareto front is also convex and connected.

The selection of a single optimum from the Pareto set needs a trade-off strategy to be implemented by the user (decision maker). A possible selection can be a point of the Pareto-front which is closest to the ideal point represented by $\left(f_{i}=0\right)$ for all $i$.

\section{The multi-objective problem}

Let us consider the Gauss-Helmert model of a weighted 2D similarity transformation problem,

$$
\left(\begin{array}{l}
X \\
Y
\end{array}\right)=F(x, y)=\left(\begin{array}{cc}
\cos \alpha & -\sin \alpha \\
\sin \alpha & \cos \alpha
\end{array}\right)\left(\begin{array}{ll}
\beta & 0 \\
0 & \beta
\end{array}\right)\left(\begin{array}{l}
x \\
y
\end{array}\right)+\left(\begin{array}{l}
\gamma \\
\delta
\end{array}\right),
$$

where $\alpha$ is the rotation angle, $\beta$ is the scale factor and $\gamma, \delta$ are the coordinates of the translation. Considering $X, Y$ and $x$, y as Cartesian coordinates of the transformed and original systems, respectively, the problem is now concerned with determining the 4 unknown parameters $(\alpha, \beta, \gamma, \delta)$ from the corresponding measured data pairs $\left\{\left(X_{i}, Y_{i}\right)\right.$, $\left.\left(x_{i}, y_{i}\right)\right\}$ having the weights $\left\{\left(W X_{i}, W Y_{i}\right)\right.$ and $\left.\left(w x_{i}, w y_{i}\right)\right\}$. It has been assumed that $x_{i}$ and $y_{i}$ are considered as independent. The following multi-objective problems can be based on the sum of squares of the local residuals:

a) In case of the transformation $(x, y) \rightarrow(X, Y)$ given by

$$
f_{1}(\alpha, \beta, \gamma, \delta)=\sum_{i=1}^{n} f_{1 i}(\alpha, \beta, \gamma, \delta)
$$

where

$$
\begin{aligned}
f_{1 i}(\alpha, \beta, \gamma, \delta)= & W X_{i}\left(X_{i}-\beta\left(\cos (\alpha) x_{i}-\sin (\alpha) y_{i}\right)+\gamma\right)^{2} \\
& +W Y_{i}\left(Y_{i}-\beta\left(\sin (\alpha) x_{i}+\cos (\alpha) y_{i}\right)+\delta\right)^{2} .
\end{aligned}
$$

b) The inverse transformation $(X, Y) \rightarrow(x, y)$ is given by

$$
\left(\begin{array}{l}
x \\
y
\end{array}\right)=F^{-1}(X, Y)=\left(\begin{array}{cc}
1 / \beta & 0 \\
0 & 1 / \beta
\end{array}\right)\left(\begin{array}{cc}
\cos \alpha & \sin \alpha \\
-\sin \alpha & \cos \alpha
\end{array}\right)\left(\begin{array}{l}
X-\gamma \\
Y-\delta
\end{array}\right)
$$

and a similar objective,

$$
f_{2}(\alpha, \beta, \gamma, \delta)=\sum_{i=1}^{n} f_{2 i}(\alpha, \beta, \gamma, \delta)
$$

where

$$
\begin{aligned}
f_{2 i}(\alpha, \beta, \gamma, \delta)= & w x_{i}\left(x_{i}-\frac{\cos (\alpha)(X-\gamma)+\sin (\alpha)(Y-\delta)}{\beta}\right)^{2} \\
& +w y_{i}\left(y_{i}-\frac{\cos (\alpha)(Y-\gamma)-\sin (\alpha)(X-\gamma)}{\beta}\right)^{2} .
\end{aligned}
$$


The multiobjective problem is to find the minimum of the competing objectives $f_{1}$ and $f_{2}$ in the design space - in our case $(\alpha, \beta, \gamma, \delta) \in R^{4}-$ in the sense of Pareto optimality.

To solve the multiobjective optimization problem, the multiobjective genetic algorithm (GA) implemented as a built-in function in Matlab as (gamultiobj) is applied.

In order to improve the efficiency of the procedure a hybrid scheme is used to find an optimal Pareto front for our multiobjective problem. The function gamultiobj can reach the region near an optimal Pareto front relatively quickly, but it can take many function evaluations to achieve convergence. A commonly used technique is to run gamultiobj for a small number of generations to get near an optimum front. Then the solution from gamultiobj is used as an initial point for another optimization solver that is faster and more efficient for a local search. Here fgoalattain is employed as the hybrid solver with gamultiobj.

One of the main advantages of this genetic hybrid algorithm is the parallelization, the parallel evaluation of the genetic algorithm - especially in our days, when multicore processors are easily available.

\section{Numerical Example}

For the numerical computation the data from Akyilmaz (2007) is employed. Corresponding coordinates of the two systems can be seen in Table I and the weights are given in Table II. Fig. 1 shows the Pareto-front of our multiobjective minimization problem.

To ensure reliable results the following parameters were used in the gamultiobj function, error limit TolFun $=10^{-12}$, limit for the number of generations StallGenLimit $=1000$ and the number of the individuals of the population PopulationSize $=300$. The computed Pareto-front consisted of 127 points. A single optimum can be selected by considering

$$
\min _{\alpha, \beta, \gamma, \delta} \sqrt{f_{1}(\alpha, \beta, \gamma, \delta)+f_{2}(\alpha, \beta, \gamma, \delta)},
$$

where $f_{1}$ and $f_{2}$ are the coordinates of a point of the Pareto-front. Practically, this optimal point is the closest point of the Pareto-front to the ideal point $(0,0)$ in the sense of the $L_{1}$ norm. The corresponding point of the Pareto-set $(\alpha, \beta, \gamma, \delta)$ can be considered as the selected Pareto solution. The selected optimum point of the Paretofront as the minimum of $f_{1}+f_{2}$ can be seen on Fig. 2 .

The parameters belonging to the selected point of the Pareto-front and the coordinates of the corresponding point of the Pareto-set are presented in Table III.

\section{Comparing Pareto optimality to other approaches}

Let us compare our results to those of the other approaches into the solution of the EIV problems.

\subsection{Ordinary least squares solutions}


a) In case of the transformation $(x, y) \rightarrow(X, Y)$ minimizing Eq. (11), we get the parameters, displayed in Table IV.

b) The inverse transformation $(X, Y) \rightarrow(x, y)$ minimizing Eq. (14), leads to the parameters, shown in Table V.

\subsection{Total least squares solution}

The TLS approach leads to a constrained minimization problem. The objective function is the sum of the weighted squares of the adjusted values of the measured variables,

$$
F=\sum_{i=1}^{n} W X_{i} \Delta X_{i}^{2}+\sum_{i=1}^{n} W Y_{i} \Delta Y_{i}^{2}+\sum_{i=1}^{n} w x_{i} \Delta x_{i}^{2}+\sum_{i=1}^{n} w y_{i} \Delta y_{i}^{2},
$$

under the constraints of the model equations,

$$
\begin{array}{ll}
g_{1 i}\left(\alpha, \beta, \gamma, \Delta X_{i}, \Delta x_{i}, \Delta y_{i}\right)=0, & i=1,2, \ldots, n, \\
g_{2 i}\left(\alpha, \beta, \delta, \Delta Y_{i}, \Delta x_{i}, \Delta y_{i}\right)=0, & i=1,2, \ldots, n,
\end{array}
$$

where

$$
\begin{aligned}
& g_{1 i}\left(\alpha, \beta, \gamma, \Delta X_{i}, \Delta x_{i}, \Delta y_{i}\right)=X_{i}+\Delta X_{i}-\beta\left(\cos (\alpha)\left(x_{i}+\Delta x_{i}\right)-\sin (\alpha)\left(y_{i}+\Delta y_{i}\right)\right)+\gamma, \\
& g_{2 i}\left(\alpha, \beta, \delta, \Delta Y_{i}, \Delta x_{i}, \Delta y_{i}\right)=Y_{i}+\Delta Y_{i}-\beta\left(\sin (\alpha)\left(x_{i}+\Delta x_{i}\right)+\cos (\alpha)\left(y_{i}+\Delta y_{i}\right)\right)+\delta .
\end{aligned}
$$

Here $\Delta X_{i}, \Delta Y_{i}, \Delta x_{i}$ and $\Delta y_{i}$, the adjusted values of the output as well as the input variables are also unknowns besides the four model parameters $(\alpha, \beta, \gamma, \delta)$.

Neitzel (2010) has solved this problem by employing the iteratively linearized least squares technique. The result can be seen in Table VI.

Since the model is linear, $\Delta X_{i}$ and $\Delta Y_{i}$ can be expressed from Eqs (20) and (21) and can be substituted into Eq. (17). In this way the constrained optimization problem can be transformed into an unconstrained one and the number of unknowns can be reduced from $4 n+4$ down to $2 n+4$.

\subsection{Least geometric mean deviation approach}

Employing the LGMD technique suggested by Tofallis (2002) for a single variable problem, one needs to consider

$$
G(\alpha, \beta, \gamma, \delta)=\sum_{i=1}^{n} \sqrt{f_{1 i}(\alpha, \beta, \gamma, \delta) f_{2 i}(\alpha, \beta, \gamma, \delta)},
$$

which corresponds to Eq (7), see Eqs. (12) and (15). The results of this minimization can be found in Table VII. The resulted values of the parameters are very close to the values given by the IL - TLS as well as Paretro optimum method.

\subsection{Comparison}

The results of these techniques are compared to the Pareto optimality solution in Fig. 3. According to our illustrative example, the LGMD solution is Pareto optimal, while 
the IL-TLS approach is very near to the Pareto front, therefore practically the Paretoset can represent all of these solutions. Consequently the Pareto optimality solution can be considered as a generalization of these solutions, see Fig. (3).

The selected Pareto solution is the point of the Pareto-front whose distance from the ideal point $(0,0)$ is minimum. The Pareto solution and the LGMD solution have practically the same value. The difference of the residuals representing the measure of the symmetry of the errors in the two different coordinate systems is also minimal in the case of the selected Pareto solution, see the third row in Table VIII.

Consequently, the selected Pareto solution can be considered as a single Pareto optimal solution, which has a mostly balanced minimum sum of squares of residuals. However, the user (decision maker) can independently define his/her specified tradeoff between the errors of the direct and inverse transformations by selecting another point on the Pareto-front.

\section{A real-word problem}

Let us consider the Budapest stereographic and the EOV coordinates (Unified National Projection System in Hungary (Völgyesi et al, 1996)) of 13 geodetic stations covering an area of $70 \times 70 \mathrm{~km}$ around Budapest, in Hungary. The coordinates of the same physical points are given in the systems $(x, y)$ and $(X, Y)$.

In this case no weights were applied. The different approaches were employed to estimate the parameters of the Gauss-Helmert transformation model. The minimization of the total residual was carried out with the genetic algorithm as global optimization method. The Pareto solution has been computed via a hybrid genetic multiobjective solver. The results can be seen in Table IX.

These results indicate very small deviations in the parameters estimated by the different methods although the Pareto solution produced the minimal total residual. The small differences of the residuals of the two coordinate systems $f_{1}-f_{2}$ shows that their errors are well balanced. Figs. (4) and (5) illustrate the distribution of the local error (norm of the local error vector) computed by the Pareto method in the system $(X, Y)$ and $(x, y)$, respectively.

These two figures are nearly identical indicating that the distribution of the local errors is also well balanced between the two systems. The relatively large local errors of all of the methods indicate that the Gauss-Helmert model is not properly suitable for the present geodetic transformation problem. However, this example shows that neither of the considered technique is able to cure the problem of the GH transformation in case of large distortions. To reduce the local errors considerably, a nonlinear transformation should be employed, see Völgyesi et al. (1996).

Fig. 6 illustrates the adjusted vectors of the TLS solution. The arrows-vectors represent the corrections (adjustments) of the measured coordinates. This figure shows that the local error of the Pareto approach and the adjustments of the TLS model are in good harmony, since where the local error of the selected Pareto solution is large, there the TLS solution provides large adjusted values.

In this sense, the Pareto approach - as a generalization of the error models discussed here - can be a good candidate for the parameter estimation of geodetic datum transformations. 


\section{Conclusions}

According to the results there is no significant difference between the selected Pareto solution and the extended LGMD method, although the latter requires less computation power than the Pareto solution. We should stress that none of these methods strictly satisfies the model equations, therefore they are able to take modeling inconsistencies into account to a certain extent. If the model is proved to be perfectly adequate then the TLS solution taking only measurement errors into account is more reasonable, since it satisfies the model equations and provides the necessary adjustments of the coordinates in both systems. However, the in case of real world problems, to use the TLS method requires global minimization with $4 n+m$ variables. In our case, the number of the variables was $4 \times 13+4=56$, which could be reduced by utilizing the linearity of the transformation down to $2 \times 13+4=30$.

\section{Acknowledgements}

The authors thank Prof. Bernhard Heck and Dr. Kevin Fleming for proof reading the manuscript, but accept all responsibility for any errors. J.L. Awange acknowledges the financial support of the Alexander von Humbold Foundation (Ludwig Leichhardt Memorial Fellowship) and a Curtin Research Fellowship. He is grateful for the warm welcome and conducive working atmosphere provided by his host, Prof. Bernhard Heck and his team, at the Geodetic Institute, Karlsruhe Institute of Technology (KIT). This work was partially funded by OTKA project No. 76231.

\section{References}

Akylmaz O 2007: Total least squares solution of coordinate transformation. Survey Review, 39(303), 68-80.

Felus Y A, Schaffrin B 2005: Performing Similarity Transformations Using the Errors-In-Variable Model. ASPRS Ann. Conference, Baltimore, Maryland.

Golub GH, van Loan C F 1980: An analysis of the total least-squares problem. SIAM J Numer Anal., 17(6): 883-893.

Marler RT, Arora J S 2004: Survey of multi-objective optimization methods for engineering, Struct Multidisc Optim 26, 369-395.

Neitzel F 2010: Generalization of total least-squares on example of weighted 2D similarity transformation. J Geodesy, DOI 10.1007/s00190-010-0408-0.

Tofallis C 2002: Model fitting for multiple variables by minimizing the geometric mean deviation. In: Van Hufferl S., Lemmerling P. (Eds.), Total Least Squares and Errors-in-Variables Modeling: Algorithms, Analysis and Applications. Kluwer Academic Publishers, Dordrecht.

Tofallis C 2003: Multiple Neutral Data Fitting. Annals Operations Research, 124, 6979.

Völgyesi L, Tóth Gy, Varga J 1996: Conversion between Hungarian Map Projection Systems. Periodica Polytechnica Civ.Eng., 40(1), 73-83. 


\section{Table Captions}

Table I: Corresponding coordinates of the two systems

Table II: Weights of the measured coordinates of the two systems

Table III: Results of the Pareto optimality computation

Table IV: Results of the Ordinary Least Squares solution in case $(x, y) \rightarrow(X, Y)$

Table V: Results of the Ordinary Least Squares solution in case $(X, Y) \rightarrow(x, y)$

Table VI: Iteratively Linearized Total Least Squares solution, Neitzel (2010)

Table VII: Results of the Least Geometric Mean Deviation approach

Table VIII: Norm and the difference of the objective functions

Table IX: Resulting parameters of the Pareto solution 
Table I. Corresponding coordinates of the two systems

\begin{tabular}{cccc}
\hline$X[\mathrm{~m}]$ & $Y[\mathrm{~m}]$ & $x[\mathrm{~m}]$ & $y[\mathrm{~m}]$ \\
\hline 4540134.278 & 382379.896 & 4540124.094 & 382385.998 \\
4539937.389 & 382629.787 & 4539927.225 & 382635.869 \\
4539979.739 & 381951.478 & 4539969.567 & 381957.570 \\
4540326.461 & 381895.009 & 4540316.294 & 381901.093 \\
4539216.387 & 382184.435 & 4539206.211 & 382190.528 \\
\hline
\end{tabular}


Table II. Weights of the measured coordinates of the two systems

\begin{tabular}{rrrr}
\hline \multicolumn{1}{c}{$W X$} & \multicolumn{1}{c}{$W Y$} & $w x$ & \multicolumn{1}{c}{$w y$} \\
\hline 10.0000 & 14.2857 & 5.8824 & 12.5000 \\
0.8929 & 1.4286 & 0.9009 & 1.7241 \\
7.1429 & 10.0000 & 7.6923 & 16.6667 \\
2.2222 & 3.2259 & 4.1667 & 6.6667 \\
7.6923 & 11.1111 & 8.3333 & 16.6667 \\
\hline
\end{tabular}


Table III. Results of the Pareto optimality computation

\begin{tabular}{cc}
\hline & Selected Pareto optimum \\
\hline$\alpha[\mathrm{rad}]$ & -0.0000039722 \\
$\beta$ & 0.9999951685 \\
$\gamma[\mathrm{m}]$ & 30.593 \\
$\delta[\mathrm{m}]$ & 13.786 \\
$f_{1}\left[\mathrm{~m}^{2}\right]$ & 0.002200 \\
$f_{2}\left[\mathrm{~m}^{2}\right]$ & 0.002332 \\
\hline
\end{tabular}


Table IV. Results of the Ordinary Least Square solution in case $(x, y) \rightarrow(X, Y)$

\begin{tabular}{cc}
\hline & OLS $(x, y) \rightarrow(X, Y)$ \\
\hline$\alpha[\mathrm{rad}]$ & -0.0000058613 \\
$\beta$ & 0.9999970178 \\
$\gamma[\mathrm{m}]$ & 21.475 \\
$\delta[\mathrm{m}]$ & 21.654 \\
$f_{1}\left[\mathrm{~m}^{2}\right]$ & 0.002113 \\
$f_{2}\left[\mathrm{~m}^{2}\right]$ & 0.002610 \\
\hline
\end{tabular}


Table V. Results of the Ordinary Least Square solution in case $(X, Y) \rightarrow(x, y)$

\begin{tabular}{cc}
\hline & OLS $(X, Y) \rightarrow(x, y)$ \\
\hline$\alpha[\mathrm{rad}]$ & -0.0000024465 \\
$\beta$ & 0.9999937289 \\
$\gamma[\mathrm{m}]$ & 37.709 \\
$\delta[\mathrm{m}]$ & 7.410 \\
$f_{1}\left[\mathrm{~m}^{2}\right]$ & 0.002419 \\
$f_{2}\left[\mathrm{~m}^{2}\right]$ & 0.002256 \\
\hline
\end{tabular}


Table VI. Iteratively Linearized Total Least Square solution, Neitzel (2010)

\begin{tabular}{cr}
\hline & \multicolumn{1}{c}{ IL - TLS } \\
\hline$\alpha[\mathrm{rad}]$ & -0.0000042050 \\
$\beta$ & 0.9999953579 \\
$\gamma[\mathrm{m}]$ & 29.643 \\
$\delta[\mathrm{m}]$ & 14.769 \\
$f_{1}\left[\mathrm{~m}^{2}\right]$ & 0.002174 \\
$f_{2}\left[\mathrm{~m}^{2}\right]$ & 0.002401 \\
\hline
\end{tabular}


Table VII. Results of the Least Geometric Mean Deviation approach

\begin{tabular}{cr}
\hline & \multicolumn{1}{c}{ LGMD } \\
\hline$\alpha[\mathrm{rad}]$ & -0.0000041015 \\
$\beta$ & 0.9999952510 \\
$\gamma[\mathrm{m}]$ & 30.168 \\
$\delta[\mathrm{m}]$ & 14.341 \\
$f_{1}\left[\mathrm{~m}^{2}\right]$ & 0.002193 \\
$f_{2}\left[\mathrm{~m}^{2}\right]$ & 0.002340 \\
\hline
\end{tabular}


Table VIII. Norm and the difference of the objective functions

\begin{tabular}{lcc}
\hline & $\sqrt{f_{1}+f_{2}} \times 10^{3}$ & $\left|f_{1}-f_{2}\right| \times 10^{4}$ \\
\hline OLS $(x, y) \rightarrow(X, Y)$ & 3.3581 & 4.97 \\
OLS $(X, Y) \rightarrow(x, y)$ & 3.3077 & 1.63 \\
IL-TLS & 3.2390 & 2.27 \\
LGMD & 3.2070 & 1.47 \\
Selected Pareto optimum & 3.2060 & 1.32 \\
\hline
\end{tabular}


Table IX. Resulting parameters of the Pareto solution

\begin{tabular}{cc}
\hline$\alpha[\mathrm{rad}]$ & 0.000063492 \\
$\beta$ & 0.999931677 \\
$\gamma[\mathrm{m}]$ & 649999.949 \\
$\delta[\mathrm{m}]$ & 238104.852 \\
$f_{1}\left[\mathrm{~m}^{2}\right]$ & 3.2329 \\
$f_{2}\left[\mathrm{~m}^{2}\right]$ & 3.2333 \\
$\sqrt{f_{1}+f_{2}}[\mathrm{~m}]$ & 4.5723 \\
$\left|f_{1}-f_{2}\right|\left[\mathrm{m}^{2}\right]$ & 0.0004 \\
\hline
\end{tabular}




\section{Figure Captions}

Fig. 1:. The Pareto-front

Fig. 2: The selected optimum point of the Pareto-front as the minimum of $f_{1}+f_{2}$

Fig. 3: Results of the different approaches: TLS( $\boldsymbol{\Delta})$, LGMD ( $\mathbf{\square})$ and the selected Pareto $(\bullet)$ solution

Fig. 4: Contour plot of the local error in the $X-Y$ system in case of the Pareto solution.

Fig. 5: Contour plot of the local error in the $x-y$ system in case of the Pareto solution.

Fig. 6: Contour plot of the local error in the $X-Y$ system in case of the Pareto solution. The vector field illustrates the adjusted vectors $(\Delta X, \Delta Y)$ of the TLS solution 
Fig. 1.

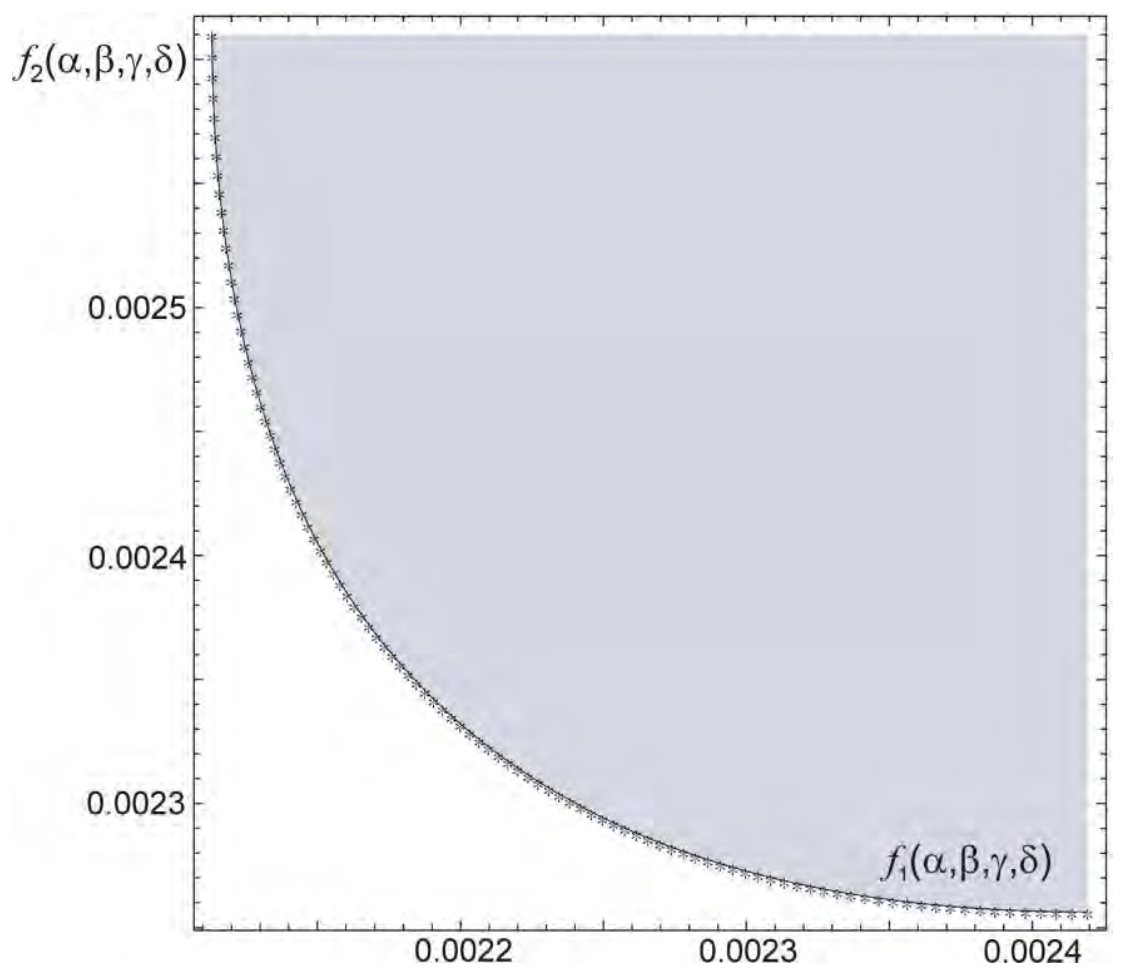


Fig. 2

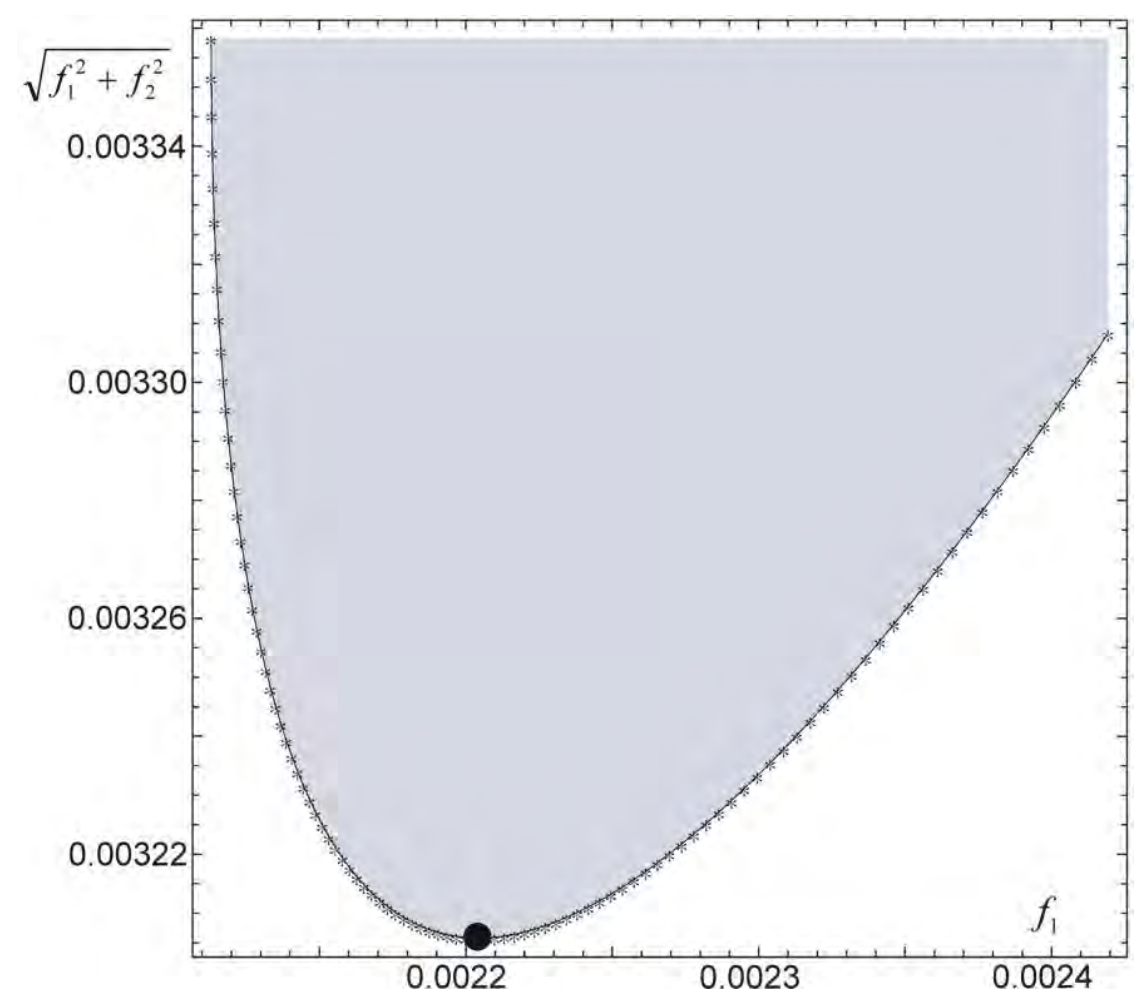


Fig. 3

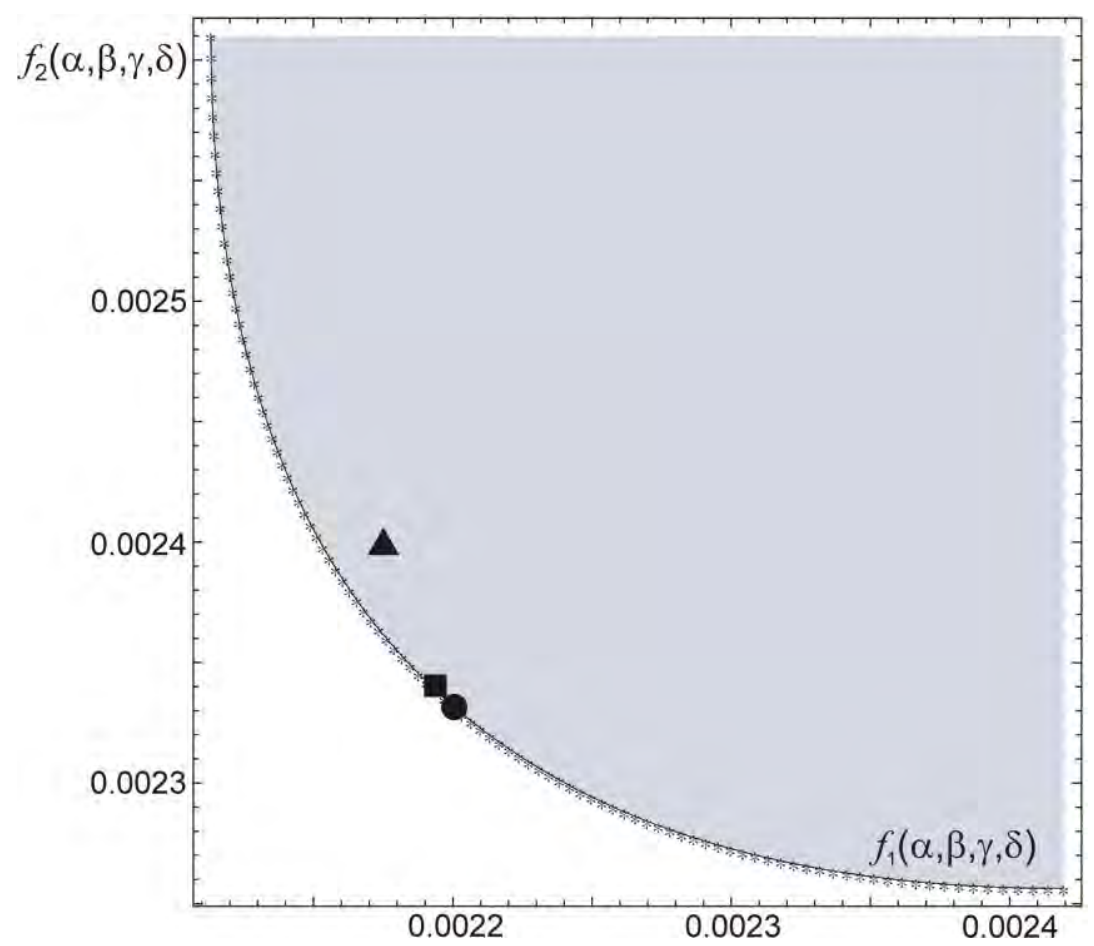


Fig. 4

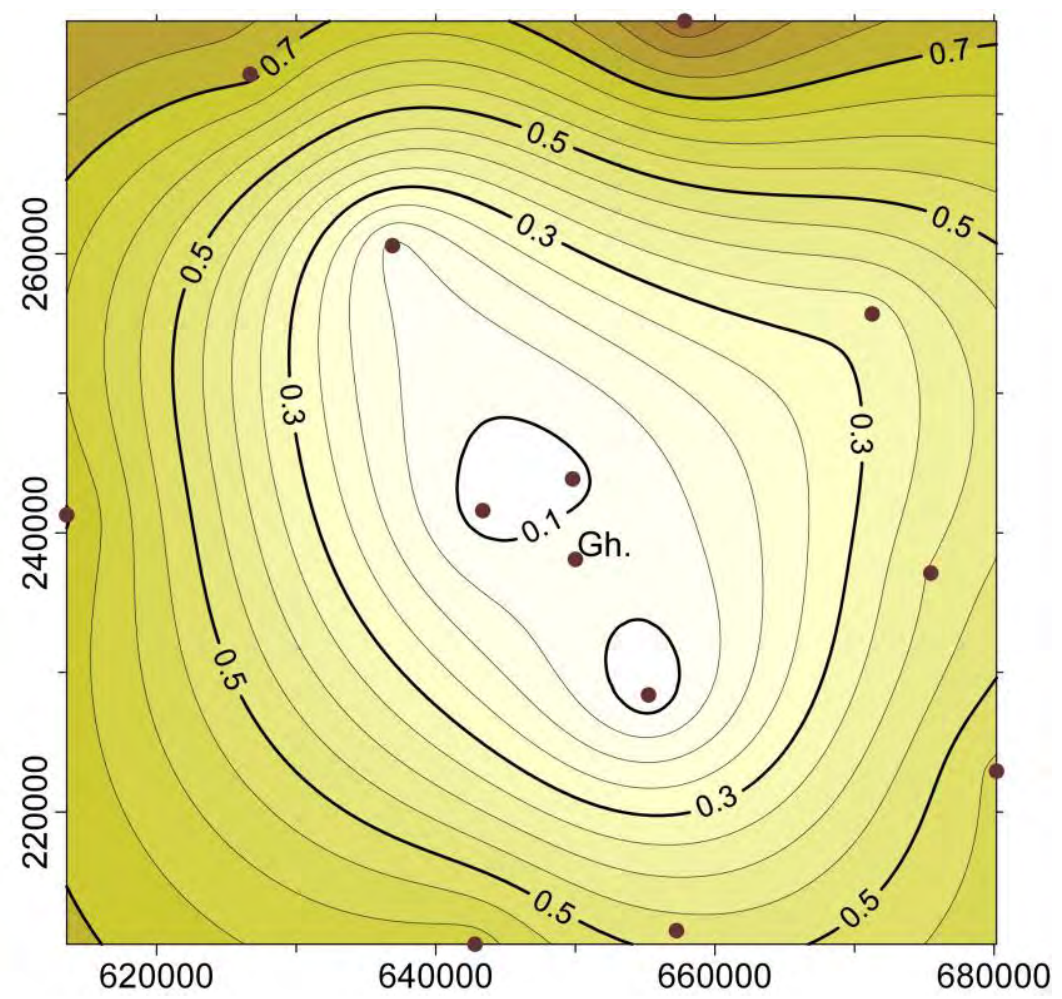


Fig. 5

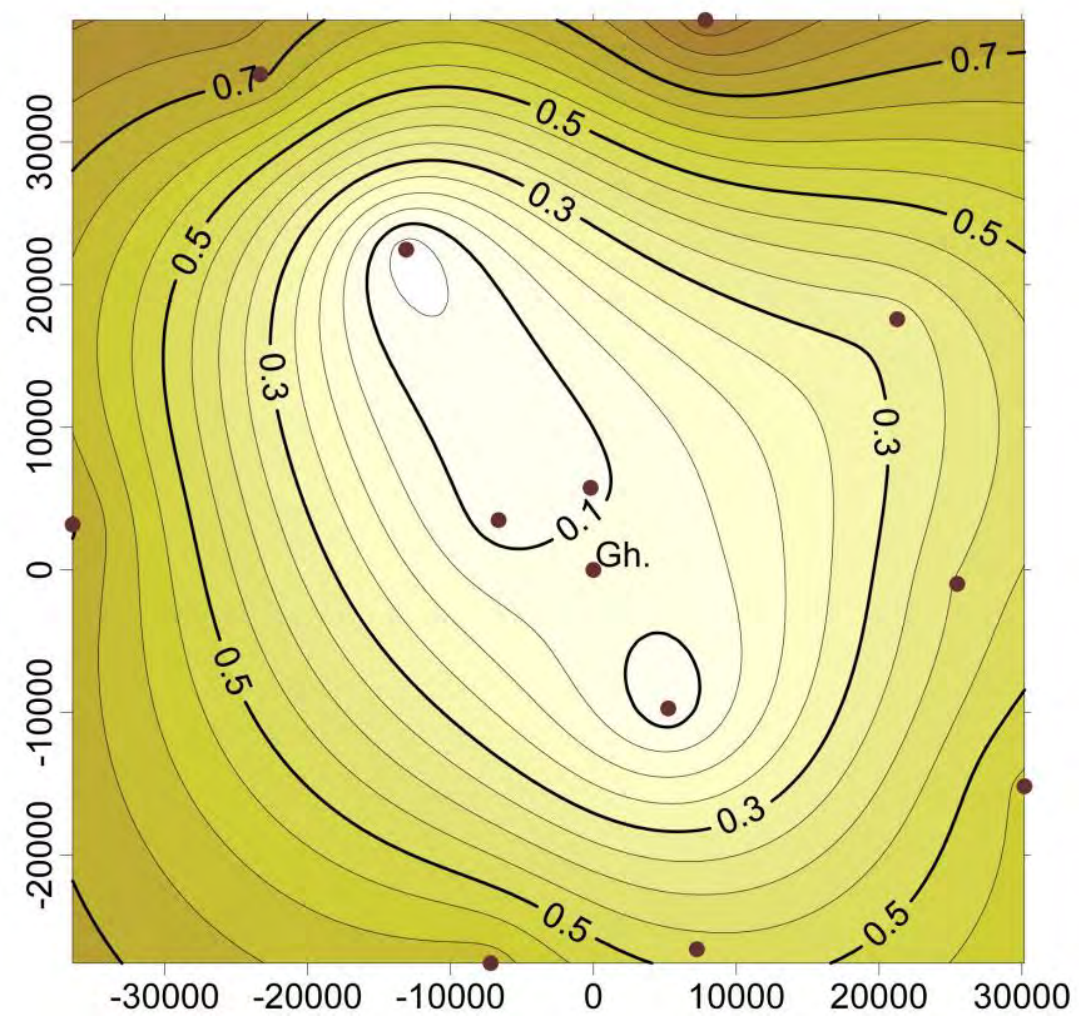


Fig. 6

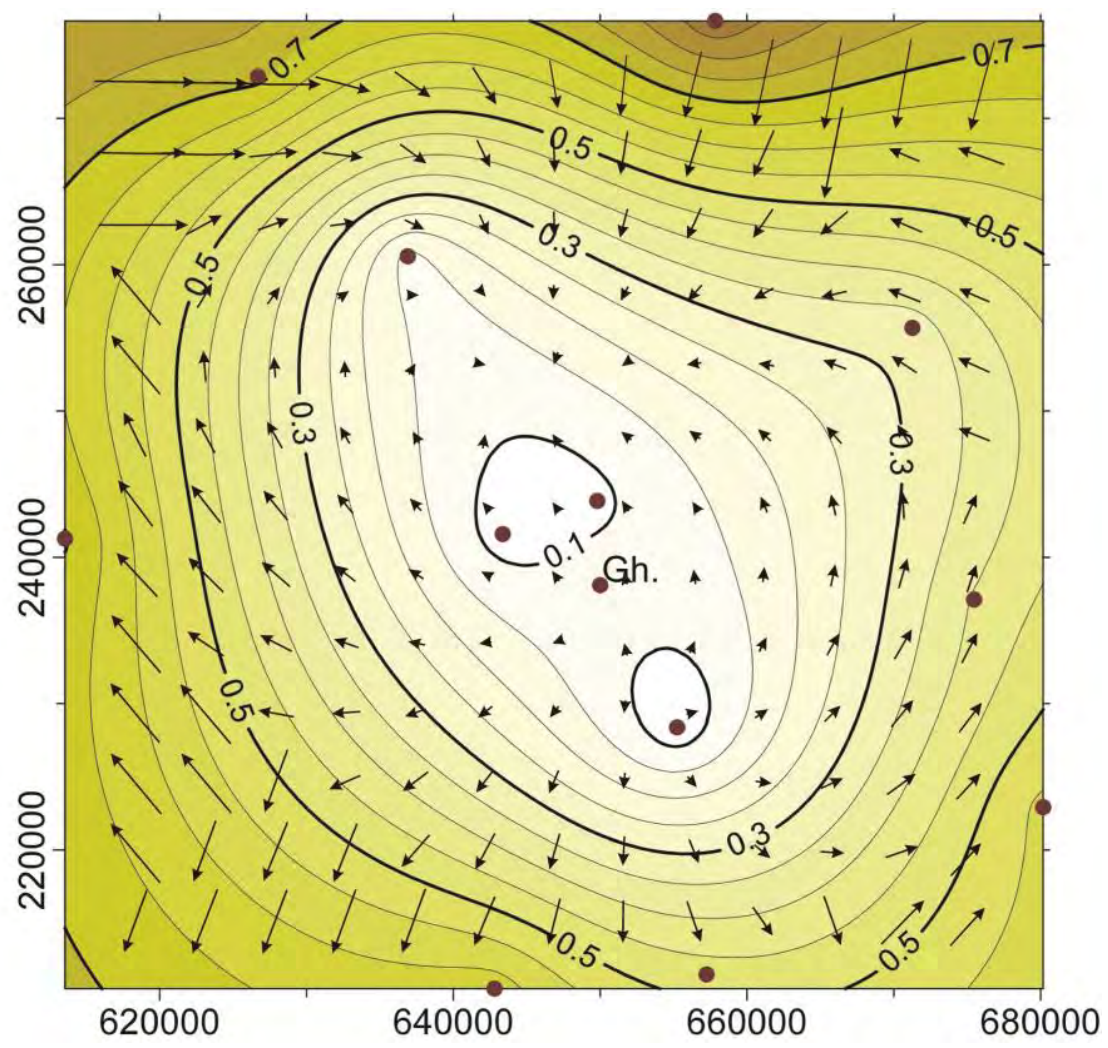

